\title{
Nature of Room-temperature Photoluminescence in $\mathrm{ZnO}$
}

\author{
W. Shan ${ }^{\mathrm{a}}$, W. Walukiewicz, J.W. Ager III, and K.M. Yu \\ Materials Sciences Division, Lawrence Berkeley National Laboratory, Berkeley, CA 94720 \\ H.B. Yuan, H.P. Xin, and G. Cantwell \\ ZN Technology, Inc. Brea, CA 92821 \\ J.J. Song \\ Department of Electrical and Computer Engineering, University of California at San Diego, La \\ Jolla, CA92093, and ZN Technology, Inc. Brea, CA 92821
}

The temperature dependence of the photoluminescence (PL) transitions associated with various excitons and their phonon replicas in high-purity bulk $\mathrm{ZnO}$ has been studied at temperatures from $12 \mathrm{~K}$ to above room temperature $(320 \mathrm{~K})$. Several strong PL emission lines associated with LO phonon replicas of free and bound excitons are clearly observed. The room temperature PL spectrum is dominated by the phonon replicas of the free exciton transition with the maximum at the first LO phonon replica. The results explain the discrepancy between the transition energy of free exciton determined by reflection measurement and the peak position obtained by the PL measurement.

PACS numbers: 78.55.Et, 78.40.Fy, 71.35.-y

$\left.{ }^{a}\right)$ Electronic mail: WShan@1bl.gov 
The fundamental properties of the wide band-gap semiconductor $\mathrm{ZnO}$ have been studied over several decades. ${ }^{1-3}$ Despite many years of extensive studies ${ }^{4-12}$ some of the fundamental properties of the luminescence in $\mathrm{ZnO}$ are still not fully understood. One of the poorly understood issues is the origin of the room temperature photoluminescence (PL) structure. Specifically, there is an energy difference between the PL peak position and the excitonic transition measured by conventional reflection or photomodulated reflectance (PR) spectroscopy. This is demonstrated in Fig. 1, where a comparison of room-temperature PL spectrum with the reflection and PR curves is given. The PL peak position at $3.25 \mathrm{eV}$ is $\sim 63 \mathrm{meV}$ lower than the excitonic transition energy of $3.313 \mathrm{eV}$ determined by the PR measurement. In addition, as is seen in Fig. 1 the PL line is asymmetric with the linewidth much larger than those of the reflection and PR spectra. These results clearly indicate that the different optical transition processes are responsible for the near band edge $\mathrm{PL}$ and reflection spectra in $\mathrm{ZnO}$ at room temperature. $^{11,12}$

In this letter, we present the results of a detailed study on temperature-dependent photoluminescence in high-purity $\mathrm{ZnO}$ bulk samples. The evolution of the emission lines arising from the radiative decay of various excitons and their phonon replicas with temperature allows us to unambiguously identify the origin of the room temperature $\mathrm{PL}$ in $\mathrm{ZnO}$.

The samples used in this work are $\mathrm{ZnO}$ bulk grown by seeded chemical vapor transport method. ${ }^{13}$ The free carrier concentration of the $\mathrm{ZnO}$ bulk prepared using this method is typically around or lower than $10^{17} \mathrm{~cm}^{-3}$. Samples were cut along the plane perpendicular to the growth direction ( $c$ axis) and polished with a chemo-mechanical method.

PL measurements were performed using the $325-\mathrm{nm}$ line from a $\mathrm{HeCd}$ laser as the excitation source. A 1-m double-grating monochromator and a UV-visible photomultiplier tube 
were used to detect the PL signals. $\mathrm{ZnO}$ samples were attached to the cold finger of a closed cycle refrigerator and cooled down to the desired temperature for measurements. For conventional reflection measurements, quasimonochromatic light from a xenon arc lamp dispersed by a $0.5-\mathrm{m}$ monochromator was focused on the samples at near-normal incidence. The reflection signals were detected by using a lock-in amplification system. A chopped $\mathrm{HeCd}$ laser beam $(325 \mathrm{~nm})$ was used to provide modulation when photomodulation measurements were conducted.

To illustrate the crystal quality of the $\mathrm{ZnO}$ samples used in this work, we show in Fig. 2 a typical reflection curve taken at $10 \mathrm{~K}$ in the energy region of the excitonic transitions. The spectrum exhibits several fine resonances corresponding to intrinsic free-exciton transitions labeled by $A(\mathrm{n}=1,2)$ at 3.3782 and $3.4216 \mathrm{eV}$, and $B(\mathrm{n}=1,2)$ at 3.3856 and $3.4273 \mathrm{eV}$, respectively. The resonances referred to as the $A$ and $B$ are excitons related to the $\Gamma_{9}{ }^{\mathrm{V}}-\Gamma_{7}{ }^{\mathrm{C}}$, and $\Gamma_{7}{ }^{\mathrm{V}}$ (upper-band) $-\Gamma_{7}{ }^{\mathrm{C}}$ interband transitions in wurtzite $\mathrm{ZnO}$, and the $\mathrm{n}=1$ and 2 are the transitions associated with the ground and the first excited states of the excitons. The spectral signature of the $C$-exciton associated with the $\Gamma_{7}^{\mathrm{V}}$ (lower-band) $-\Gamma_{7}{ }^{\mathrm{C}}$ interband transition is hardly detectable because the transition process is theoretically forbidden for the wave vector of the incident light along the $c$ axis $(\mathbf{k} \| c)$ and the polarization perpendicular to the axis $(\mathbf{E} \| c) \cdot{ }^{1,14}$ An estimate of the binding energy for the respective excitons from the separation between the $\mathrm{n}=1$ and $\mathrm{n}=2$ states yields $\sim 58$ and $\sim 56 \mathrm{meV}$ for $A$ and $B$ excitons, respectively, assuming the hydrogenic model based on the effective mass approximation is applicable.

The details of PL features related to various exciton (zero-phonon) emissions obtained at $12 \mathrm{~K}$ are shown in the inset of Fig. 2. As commonly observed in $\mathrm{ZnO}$, the near band-edge emission is dominated by radiative decays from a number of bound exciton complexes with the 
strongest neutral-donor-bound doublet peaked at 3.3617 and $3.3622 \mathrm{eV}$. The PL spectral feature from the $A$-exciton emission was barely observable. It appears at $3.378 \mathrm{eV}$ as a shoulder with an intensity almost two orders of magnitude weaker than those bound exciton emissions.

In addition to the near-band-edge exciton emissions, a series of PL spectral features could be clearly observed between $3.35-3.30 \mathrm{eV}$ as illustrated in Fig. 3. Starting from higher energy side the major spectral features include two relatively strong emission lines and two sets of equal distanced spectral features (up to five) in the PL spectra. The signal intensity of one set goes down and the other goes up as the sample temperature is raised from 12 to $70 \mathrm{~K}$. The two emission lines located next to the bound exciton emission structure with the peak energies of 3.3238 and $3.3324 \mathrm{eV}$ are most likely from radiative recombination processes arising from the donor-to-acceptor pair transitions, rather than two-electron transitions, ${ }^{7}$ because the energy separation between them is much larger and their linewidths are much broader than those of the bound excitons. Further support for this assignment is provided by the observed rapid thermal quench of the emissions at higher temperatures. ${ }^{4,5}$

Based on the observed temperature dependence and measured energy separations, the first set of the equal distanced PL features can be attributed to the LO phonon lines resulting from the simultaneous emission of photons and phonons in the annihilation process of free excitons. A general relationship for the emission lines involving phonons and the exciton emission can be written as

$$
E_{\mathrm{m}}=E_{0}-m \hbar \omega_{\mathrm{LO}}+\Delta E,
$$

where $E_{\mathrm{m}}$ denotes the energy position of the spectral peaks, $E_{0}$ is the exciton energy at $\boldsymbol{K}=0, m$ is an integer, and $\hbar \omega_{\mathrm{LO}} \approx 72 \mathrm{meV}$ is the $\mathrm{LO}$ phonon energy. ${ }^{15}$ The term $\Delta E=\hbar^{2} \boldsymbol{K}^{2} / 2 M$ is the kinetic energy of free excitons in the crystal. Assuming a Maxwellian distribution ${ }^{16,17}$ for thermalized 
excitons, one obtains that the separation of the first phonon line from the free-exciton emission is slightly less than $\hbar \omega_{\mathrm{LO}}$. It is seen from Fig. 3 that the intensity of the two series of peaks one associated with the free and the other with bound excitons exhibit opposite dependence on temperature. The intensities of the free-exciton phonon replicas increase with temperature whereas the intensities of the bound-exciton replicas decrease with temperature and are not detectable at temperatures higher than $70 \mathrm{~K}$. This effect can be primarily attributed to the change in the thermal occupation of the ground states of free and bound excitons. The fast reduction in the emission intensity is a result of the rapid thermal ionization of bound excitons with increasing temperature. ${ }^{18}$

Shown in Fig. 4 are PL spectra taken at selected higher temperatures compared to those in Fig. 3. The sharp, well resolved emission lines of the free-exciton phonon replicas are thermally broadened and evolve into an asymmetric featureless PL curve. At the same time the intensity of the bound-exciton emissions rapidly decreases. From the results of the figure it is clearly seen that the maximum of the PL spectrum coincides with the emission of $1 \mathrm{LO}$ phonon replica of the free excitons $\left(\mathrm{E}_{\mathrm{FX}}-1 \mathrm{LO}\right)$. It becomes the strongest PL feature at temperatures higher than $130 \mathrm{~K}$. As a result, the room-temperature PL peak position is lower than the free exciton energy in $\mathrm{ZnO}$ by the approximate amount of $\hbar \omega_{\mathrm{LO}}-\Delta E$, where is the energy corresponding to the maximum of the thermal occupation free excitons. At room temperature, with $\Delta E \approx k T / 2=12.5 \mathrm{meV}$, the expected shift of the PL line maximum equals to $\hbar \omega_{\mathrm{LO}}-\Delta E \approx 60$ $\mathrm{meV}$, which is close to the experimentally observed energy difference of $63 \mathrm{meV}$ between the PL peak position and the free exciton energy at room temperature. ${ }^{19}$ The asymmetric shape of the PL spectrum with a distinct low energy tail can be attributed to the contribution from the higher order phonon replicas to the emission. 
It is interesting to see that the zero-phonon free-exciton emission $(\boldsymbol{K} \approx 0)$ becomes weaker than 1LO- and 2LO-phonon assisted annihilation processes of free excitons in $\mathrm{ZnO}$ as the sample temperature is increased. This indicates that the LO-phonon-exciton coupling is very efficient in this highly polar material. The reported LO-phonon-exciton coupling constant of $\mathrm{ZnO}$ derived by Makino et al from absorption measurements is nearly four times stronger than that of $\mathrm{GaN}$ and about eight times stronger that of $\mathrm{ZnSe} .{ }^{20}$ As the average kinetic energy of the free excitons increases with temperature, the thermal redistribution results in a decreasing number of the excitons close to the center of the Brillouin-zone reducing the efficiency of the radiative recombination. As a result the simultaneous emission of photons and LO phonons becomes most efficient free exciton annihilation process. In addition, the free exciton binding energy of $\sim 60$ $\mathrm{meV}$ is nearly resonant with the $\sim 72 \mathrm{meV}$ LO-phonon energy in $\mathrm{ZnO}$ resulting in additional enhancement of the electron phonon interaction.

In conclusion, the effect of temperature on the PL spectra of free- and bound-excitons and their phonon replicas in $\mathrm{ZnO}$ has been studied in detail. The results demonstrate that the room temperature $\mathrm{PL}$ of $\mathrm{ZnO}$ is dominated by the free exciton $\mathrm{LO}$ phonon replicas emissions with the maximum located close to $\mathrm{E}_{\mathrm{FX}}-1 \mathrm{LO}$ energy. Our results explain the discrepancy between the transition energy of free exciton determined by conventional reflection and PR measurements and the emission peak position measured by PL at room temperature.

This work at LBNL is supported by the Director, Office of Science, Office of Basic Energy Sciences, Division of Materials Sciences and Engineering, of the U.S. Department of Energy under Contract No. DE-AC03-76SF00098. 


\section{References}

1. D.G. Thomas, J. Phys. Chem. Solids, 15, 86(1960).

2. W.Y. Liang and A.D. Joffe, Phys. Rev. Lett. 20, 59(1968).

3. K. Hümmer, Phys. Status Solidi, 56, 249(1973).

4. E. Tomzig and R. Helbig, J. Lumin. 14, 403(1974).

5. Ch. Solbrig and E. Mollwo, Solid State Commun. 5, 625(1975).

6. J. Gutowski, N. Presser, and Broser, Phys. Rev. B38, 9746(1988).

7. D.C. Reynolds, D.C. Look, B. Jogai, C.W. Litton, T.C. Collins, W. Harsch, and G. Cantwell, Phys. Rev. B57, 12151(1998).

8. D.M. Bagnall, Y.F. Chen, Z. Zhu, T. Yao, M.Y. Shen, and T. Goto, Appl. Phys. Lett. 73, 1038(1998).

9. D.C. Reynolds, D.C. Look, B. Jogai, and J.E. Hoelscher, R.E. Sherriff, M.T. Harris, and M.J. Callahan, J. Appl. Phys. 88, 2152(2000).

10. N. Ohashi, T. Sekiguchi, K. Aoyama, T. Ohgaki, Y. Terada, I. Sakaguchi, T. Tsurumi, and H. Haneda, J. Appl. Phys. 91, 3658(2002).

11. D.W. Hamby, D.A. Lucca, M.J. Klopfstein, G. Cantwell, J. Appl. Phys. 93, 3214(2003).

12. L. Wang and N.C. Giles, J. Appl. Phys. 94, 973(2003).

13. G. Cantwell, H.P. Xin, H.B. Yuan, J.J. Song, C.W. Litton, Y.K. Yeo and T. Steiner, The Third International Workshop on ZnO and Related Materials, Oct. 5-8, 2004, Sendai, Japan.

14. D.G. Thomas and J.J. Hopfield, Phys. Rev. 116, 573(1959).

15. Landolt-Börstein, edited by O. Madelung, New series, Group III, Vol.17b (Springer, Berlin, 1982); Vol.22a (1988).

16. E. Gross, S. Permogorov, and B. Razbirin, J. Phys. Chem. Solids, 27, 1647(1966). 
17. H.B. Bebb and E.W. Williams, in Semiconductors and Semimetals, ed. R.K. Willardson and A.C. Beer, (Academic, New York, 1972), Vol.8, p.182.

18. W. Shan, X.C. Xie, J.J. Song, and B. Goldenberg, Appl. Phys. Lett. 67, 2512(1995).

19. M. Ueta, H. Hanzaki, K. Kobayashi, Y. Toyozawa, and E. Hanamura, Excitonic Processes in Solids (Springer, Berlin, 1986).

20. T. Makino, C.H. Chia, N.T. Tuan, Y. Segawa, M. Kawasaki, A. Ohtomo, K. Tamura, and H. Koinuma, Appl. Phys. Lett. 76, 3549(2000). 


\section{Figure Captions:}

Figure 1. Comparison of PL spectrum with conventional reflection and $\mathrm{PR}$ curves of $\mathrm{ZnO}$ recorded at $295 \mathrm{~K}$.

Figure 2. 10-K conventional reflection spectrum of $\mathrm{ZnO}$ bulk. The marked resonances correspond to $A$ - and $B$-exciton transitions involving the ground $(\mathrm{n}=1)$ and the first excited $(\mathrm{n}=2)$ states. The inset shows the details of near band-edge emissions at $12 \mathrm{~K}$.

Figure 3. PL spectra taken at temperatures from 12 to $70 \mathrm{~K}$. The spectra are plotted in logarithm scale and vertically displaced for clarity. The dashed lines indicate the emission lines involving phonon replicas of the free excitons and bound excitons.

Figure 4. The lineshape change of PL spectrum of $\mathrm{ZnO}$ with temperature. The spectra are

plotted in logarithm scale and vertically displaced for clarity. The distinctively sharp PL spectral featuers from phonon-replica emissions evolve into a featureless emission band with the 1LO emission at the maximum as temperature is raised. 


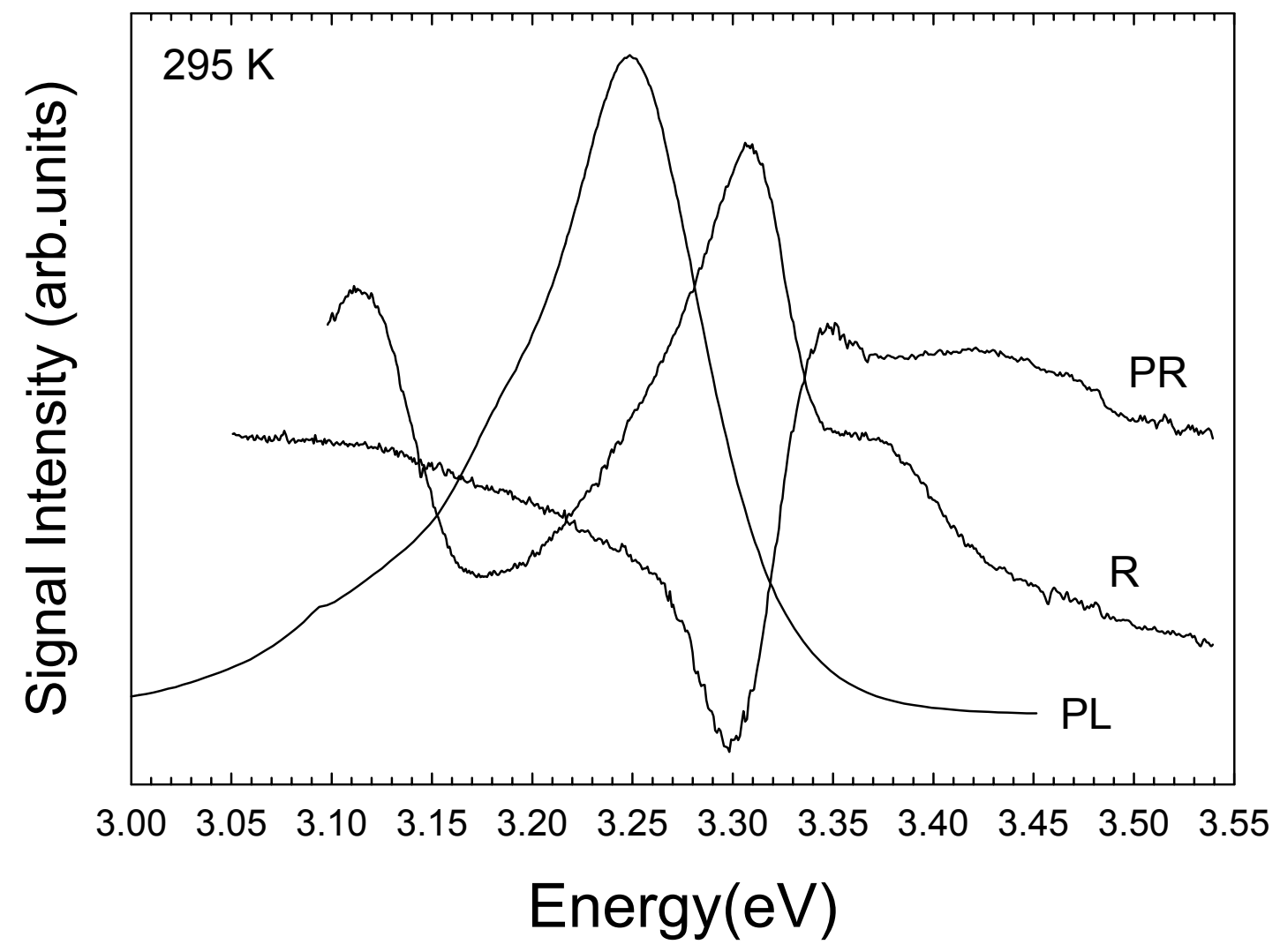

Figure 1/4, Shan et al 


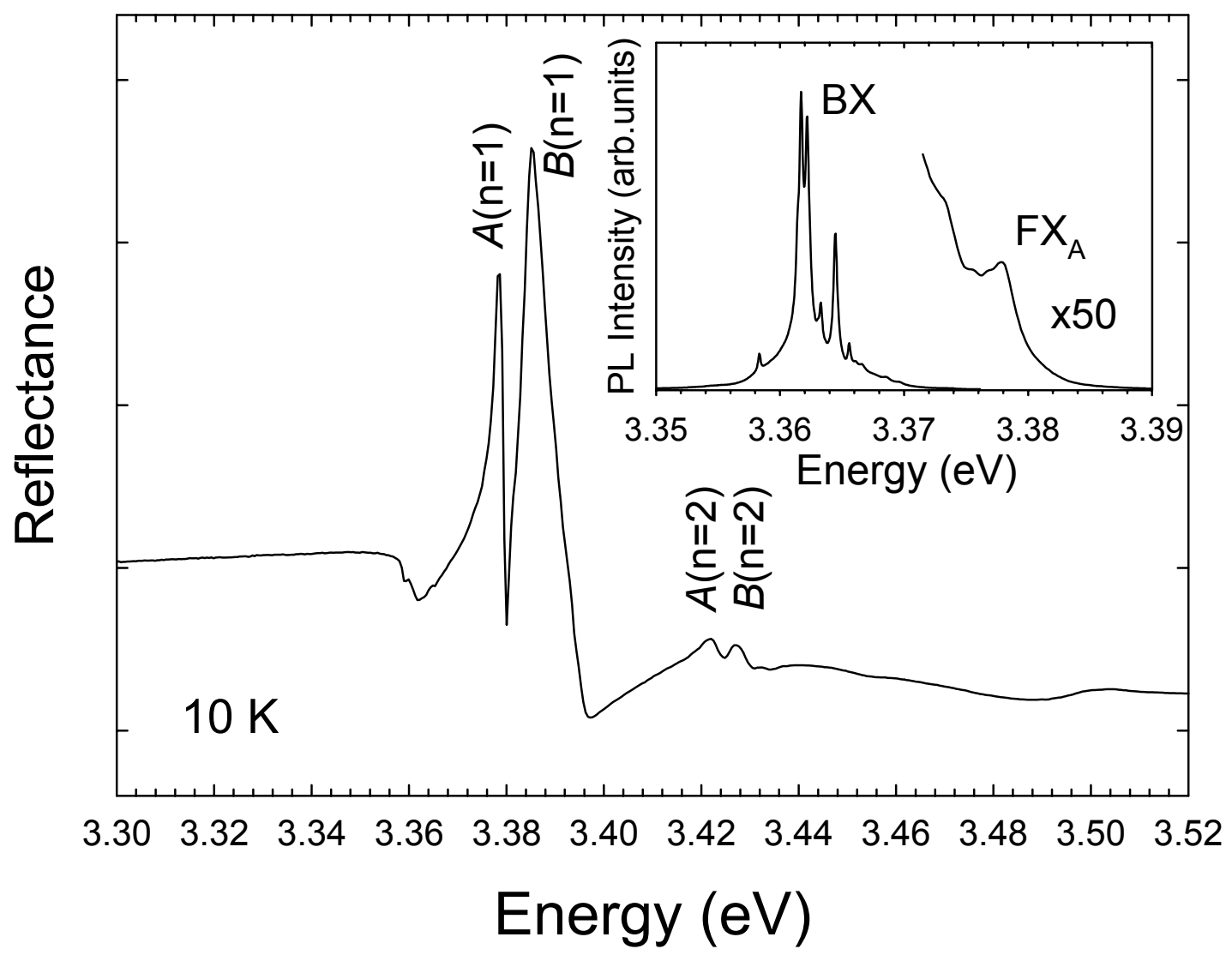

Figure 2/4, Shan et al. 


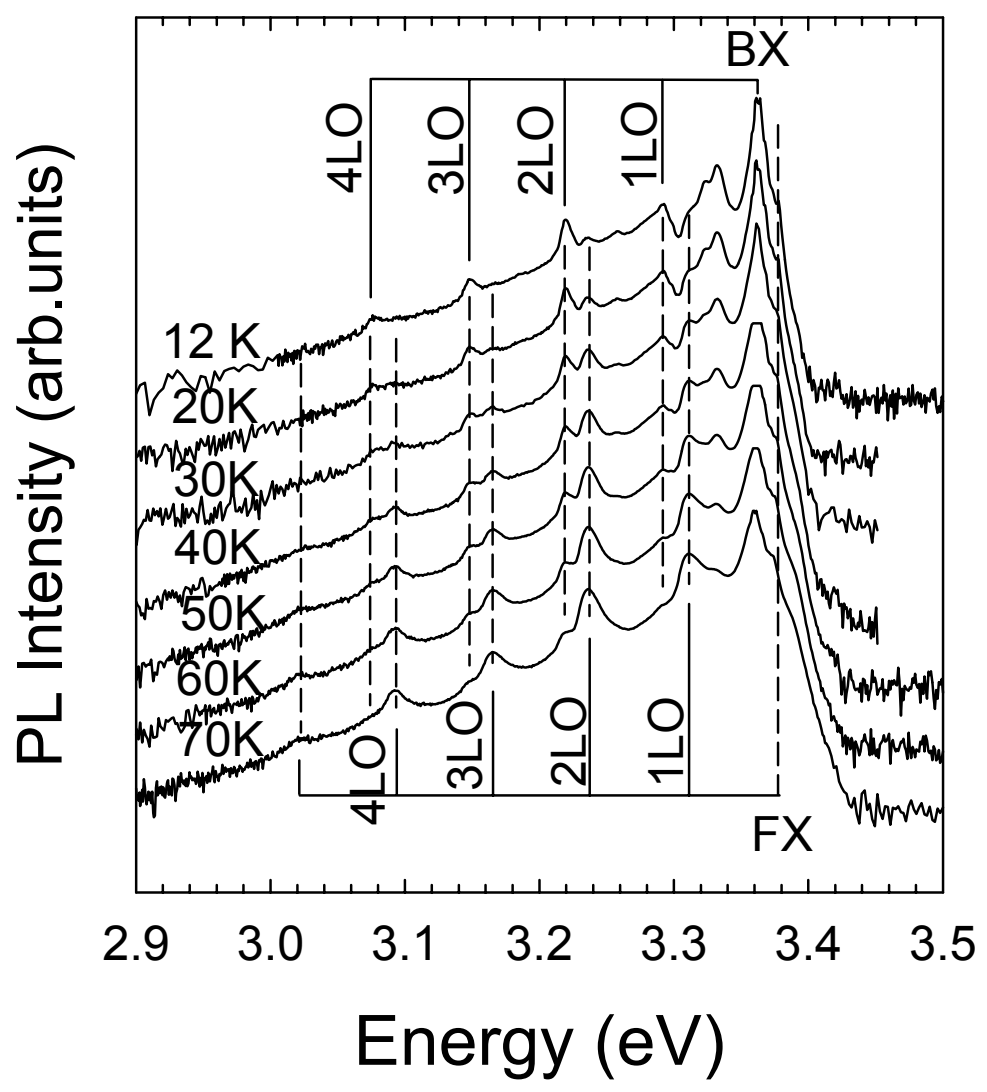

Figure 3/4, Shan et al. 


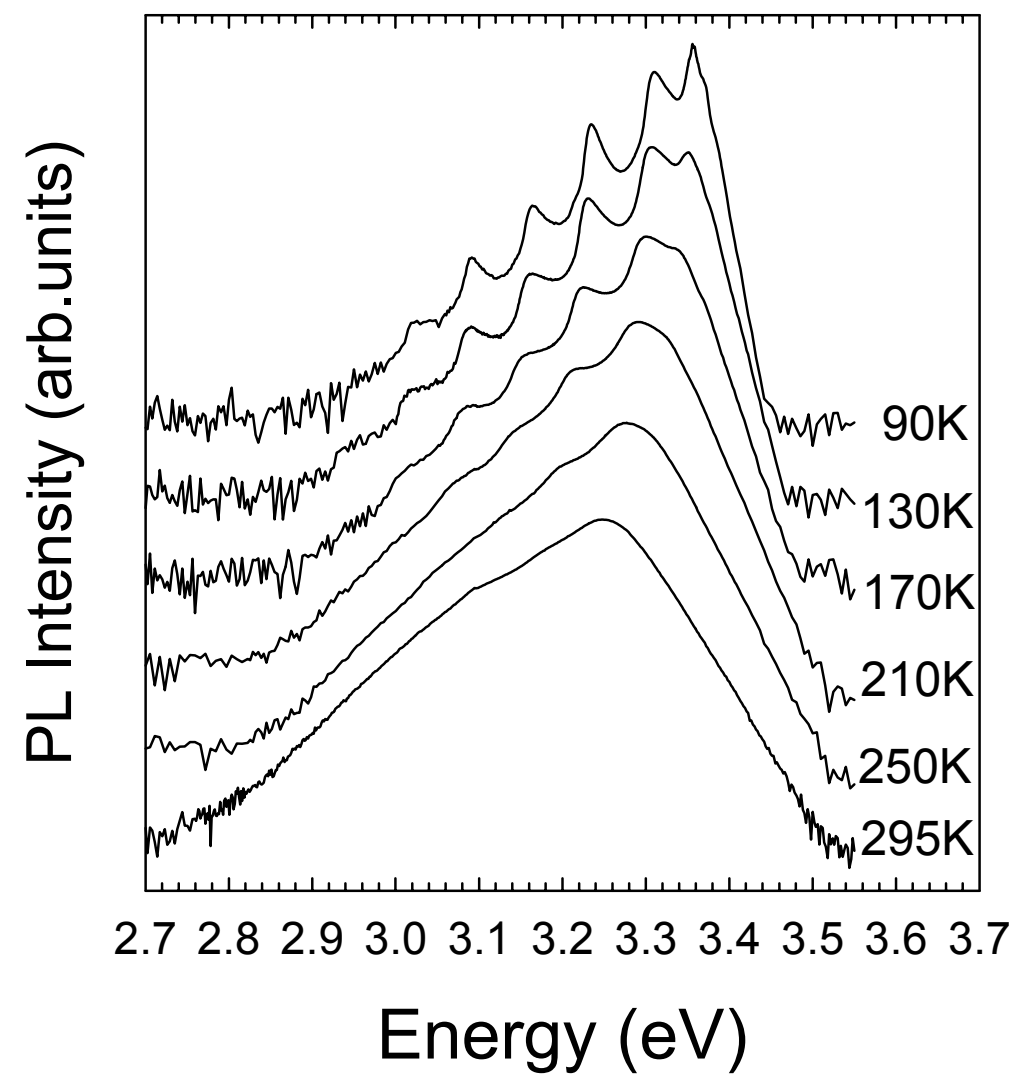

Figure 4/4, Shan et al. 